Gutiérrez, S., y Montañez, G. S. (2018). Inserción de la mujer en cargos gerenciales en la industria tequilera. Revista Lebret, 10, 73-88 - ISSN Versión impresa: 2145-5996 - ISSN Versión Web on line: 2357-5468 - https://doi.org/10.15332/rl.v0i10.2198

\title{
Inserción de la mujer en cargos gerenciales en la industria tequilera ${ }^{1}$
}

\section{Insertion of women in management positions in the tequilera industry}

\author{
Sandra Gutiérrez Olvera \\ Gloria Silviana Montañez Moya
}

\begin{abstract}
Resumen
En la presente investigación se analiza información con el objetivo de definir el perfil de la mujer gerencial y comparar las conductas de liderazgo de hombres y mujeres en las empresas tequileras de Jalisco, México. El estudio es una investigación con enfoque cuantitativo transversal y alcance descriptivo, la población son las empresas tequileras de Jalisco, se determinó entrevistar a aquellas con más de 30 trabajadores, obteniendo una muestra de 12 empresas, y al final se pudieron encuestar 8 , en donde se entrevistaron a 38 gerentes 12 mujeres y 26 hombres. Con los resultados obtenidos, se determinó un perfil de la mujer gerencial y, además, se evidencia que las conductas de líder en la mujer están orientadas hacia las tareas.
\end{abstract}

\section{Palabras clave}

Mujer gerencial, conductas de liderazgo y perfil.

Códigos de clasificación JEL: J16, M10, J20

\begin{abstract}
In the present investigation, information is analyzed with the objective of defining the profile of the managerial woman and comparing the leadership behaviors of men and women in the tequila companies of Jalisco, Mexico. The study is a research with a transversal quantitative approach and a descriptive scope, the population is the tequila companies of Jalisco, it was determined to interview those with more than 30 workers, obtaining a sample of 12 companies and in the end they could survey 8 , in where 38 managers were interviewed 12 women and 26 men. With the results obtained, a profile of the managerial woman was determined, and it is also evident that the leader behaviors in women are task-oriented.
\end{abstract}

\section{Keywords}

Managerial woman, leadership behaviors and profile.

\section{Artículo de investigación.}

2 Centro Universitario de los Valles de la Universidad de Guadalajara. Correo electrónico: sandraguvera@profesores.valles.udg.mx. Código ORCID: 0000-0003-0791-9565

3 Universidad de Guadalajara. Correo electrónico: gloriasilviana@gmail.com. Código ORCID: 0000-0002-5557-1279 


\section{Introducción}

Actualmente la mujer ha tomado mayores responsabilidades en lo familiar, social, cultural y económico (García y De Oliveira, 2006). Sin embargo, el principio de igualdad de oportunidades y de no discriminación por razón de sexo resulta solo un criterio legal, pero no un hecho plenamente conseguido. Así, aunque la incorporación de las mujeres al mercado laboral ha experimentado un extraordinario desarrollo en las últimas décadas, uno de los ejemplos más patentes de la persistencia de la desigualdad lo constituye el diferente grado de ocupación de puestos de decisión y cargos de responsabilidad por parte de hombres y mujeres en distintos ámbitos y organizaciones, hecho que, con mayor o menor incidencia, ocurre en casi todos los países.

La Carta de las Naciones Unidas, firmada en 1945, fue el primer acuerdo internacional para afirmar el principio de igualdad entre mujeres y hombres. Desde entonces, la Organización de la Naciones Unidas (ONU) ha contribuido a crear un legado histórico de estrategias, normas, programas y objetivos acordados internacionalmente para mejorar la condición de las mujeres en todo el mundo. Pese a que la participación laboral de la mujer en México ha aumentado, aún persisten diferencias en los puestos directivos y lo que ganan en comparación con los hombres.

Desde el año 1980, se ha dado un aumento de la inclusión de la mujer en el ámbito laboral en países latinoamericanos, los puestos gerenciales ocupados por las mujeres representan entre el $25 \%$ y el 35\% (ONU, 2005). Sin embargo, ni la mayor participación de las mujeres en el mercado laboral, ni las medidas legales adoptadas, ni la formación académica recibida han generado un aumento proporcional de la representación femenina en puestos gerenciales, tal como aparentemente los indicadores muestran (Cuadrado y Morales, 2007).

No obstante, de los 35 países que componen la Organización para la Cooperación y Desarrollo Económicos (OCDE) ubican a México en los últimos lugares de los países donde son menos las mujeres que ocupan puestos en consejos directivos, solo por encima de Japón, Chile y Brasil. Esto debido a que menos del 10\% de los puestos en los consejos administrativos están ocupados por mujeres. En el extremo opuesto se ubica Noruega, donde casi el $40 \%$ de las féminas tienen trabajos directivos.

Por otro lado, en México el tequila es un producto tradicional y representativo de la cultura; su industria aporta una parte significativa del PIB y es fuente de empleo en varios municipios del Estado de Jalisco (Macías, 1997). La industria tequilera se encuentra caracterizada como un oligopolio, ya que cuatro empresas controlan el $65 \%$ del mercado, donde no todas las empresas ponen énfasis en su capital humano (Coelho, 2007). Así pues, dada la importancia de este sector empresarial para la economía nacional, el presente estudio se centra en identificar la participación de la mujer en los puestos gerenciales de la industria del tequila en el estado de Jalisco. 
Por lo tanto, la importancia de la mujer en el ámbito laboral es indiscutible, y a pesar del incremento de la participación de las mujeres en el mercado laboral, su acceso a los puestos directivos y gerenciales es limitado, y se debe valorar la transcendencia de la inclusión de las mujeres en cargos directivos, porque aumentaría la pluralidad de enfoques y de puntos de vista en las organizaciones.

\section{Diversidad de género}

En los últimos años, el análisis de las relaciones de género en el mundo laboral se ha interesado por: a. Describir las prácticas de discriminación laboral entre hombres y mujeres, b. Desarrollar teorías sobre barreras de acceso y promoción de la mujer al mundo laboral y c. Desarrollar propuestas de cambio y líneas de intervención afines a los hitos planteados. (Selva, Sahagún y Pallares, 2011, p. 228).

Es necesario diferenciar el sexo de género, puesto que el primero es un término biológico, en cambio el segundo es un concepto amplio, que establece papeles y valores relacionados con cada uno, considerando aspectos históricos y sociales (Gálvez, 2001).

Las personas no solo desarrollan creencias sobre los hombres y las mujeres en general, sino que también generan autopercepciones o creencias de género sobre sí mismas. El concepto de identidad de género atribuye parte del comportamiento propio a determinados rasgos, características, roles u ocupaciones tipificadas como masculinas o femeninas (Barberá, Ramos y Candela, 2011, p. 157).

En este sentido, el género es lo que significa ser hombre o mujer, y su relación con lo masculino y lo femenino; así mismo, cómo este hecho define las oportunidades, los papeles, las responsabilidades y las relaciones entre las personas, dentro de una comunidad u organización (García, González, y Murillo, 2017). Si se asume que hombres y mujeres son distintos, el liderazgo, definido como una serie de comportamientos que implican la comunicación, la forma en que se administran los conflictos, la toma de decisiones y demás, traerá consigo ciertas particularidades en razón de tales diferencias (Contreras, Pedraza y Mejía, 2012, p. 188).

Las diferencias de género en la forma de ejercer la función de dirección y liderazgo están marcadas por características propias de ser hombre o mujer. Por una parte, se considera que las mujeres muestran un comportamiento demasiado emocional, tienen menos compromiso que los hombres, carecen de motivación y son menos asertivas (García, Salas y Gaviria, 2017, p. 31).

También se subraya que en comparación con los hombres, las mujeres asumen un rol más empático y se centran en la aprobación y la afiliación, lo que les permite alcanzar satisfacciones personales, por decisión propia y no por situaciones inequitativas (Clancy, 2007). 
Se afirma que las mujeres presentan mayor inclinación hacia la interrelación, al centrarse en cuestiones de afecto y sensibilidad emocional, a diferencia de los hombres que tienden más a controlar, dominar, ambicionar y ser individualistas (Eagly y Carli, 2007).

La socialización femenina hacia el cuidado de la familia y el hogar, y su escasa experiencia (tanto biográfica como histórica en estos cargos), es protagónica en su comportamiento en tanto le incita a buscar aprobación a través de modelos flexibles y amigables hacia sus subordinados (Moncayo y Zuluaga, 2015, p. 154).

Dadas las diferencias propuestas por varios autores, se plantea que la discusión en torno al género debe centrarse en el reconocimiento de tales diferencias, e incluso exaltarlas en un contexto de equidad, es decir, en un marco de oportunidades asequibles para todos. De esta manera, el que las organizaciones posean líderes hombres y mujeres, constituirá una fuente de riqueza en cuanto la diversidad en sí misma lo es (Gálvez, 2001).

En este contexto, la diversidad de género en los mandos directivos puede convertirse en una necesidad para aprovechar el potencial que ambos aportan a las organizaciones. Lo que distinguirá en el futuro a una empresa con éxito de las demás, será el uso de los recursos humanos, para lo que los hombres y las mujeres podrían tener cualidades distintas y complementarias (Berenguer, Cerver, De la Torre y Torcal, 2004, p. 126). Asumir la diversidad implicaría reconocer que las diferencias psicológicas, biológicas, sociales y culturales, intervienen en la conducta de hombres y mujeres.

\section{Conductas del liderazgo}

En el estudio de Eagly y Johnson publicado en 1990 (citado por Camps, Pérez y Martínez, 2010, p. 117) se concluye que hombres y mujeres practican un estilo de líder con inclinación hacia la tarea en el desempeño laboral en concordancia con su género. Se evidenció que las mujeres guiaban con estilos más incluyentes y colaborativos que los hombres, quienes lo ejercían de modo más unilateral que ellas.

Las teorías situacionales y de contingencia enfatizan la influencia de los contextos sobre las conductas y los estilos de liderazgo, bajo esta idea el líder resalta la necesidad de desarrollar conductas para conseguir metas organizativas en un modelo empresarial racional y predecible (Berenguer, Cerver, De la Torre, y Torcal, 2004, p. 127).

Los enfoques convencionales de liderazgo se dividen en dos: orientado a la tarea y centrado hacia las personas. El líder orientado a la tarea, realza las cuestiones técnicas laborales encaminadas a la conclusión y correcto logro de los objetivos del equipo, denominados también como líderes autoritarios. Los centrados hacia las personas, se enfocan en los trabajadores, se preocupan por sus intereses, reconocen su 
individualidad y promueven la autonomía en el desempeño de su labor (Cuadrado, Navas y Molero, 2004).

El líder transaccional asume una reciprocidad de estímulos entre el líder y los trabajadores: el líder expresa sus expectativas del personal laboral y las recompensas por el trabajo desempeñado correctamente y también sobre las sanciones por incumplir las tareas encomendadas. El líder transformacional, a diferencia, impulsa el crecimiento de los trabajadores para el logro de las metas establecidas en la organización desde la integración del grupo. Se enfoca hacia los intereses de cada uno y se transfiere a los trabajadores la necesidad de obtener los mejores resultados, impulsándolos para que participen en su logro (Charlo y Núñez, 2012, p. 91).

\section{Perfil de mujeres en puestos gerenciales}

El papel de la mujer en el entorno organizacional y gerencial ha aumentado de manera dinámica y colaborativa actualmente. No obstante, la alta integración de la mujer al campo empresarial debería también verse en su ingreso a cargos gerenciales. Este análisis se basa en el principal esquema: si se tiene un alto número de trabajadores, lo lógico es que se pueda elegir gerentes con mejor desempeño. La pluralidad debería de centrase como una ventaja competitiva y no como un requisito jurídico. Para contender en la globalización, las organizaciones requieren tener y motivar a los mejores trabajadores independientemente del género (Charlo y Núñez, 2012, p. 90).

Los hombres siguen ostentando la mayoría de los puestos directivos, altos ejecutivos y altos niveles de profesionales, mientras que las mujeres todavía se encuentran concentradas en los niveles más inferiores de la dirección. La dificultad para el acceso de las mujeres a los puestos de alta dirección no se limita solamente al mundo de la empresa, sino que es una constante en todas las áreas profesionales (De Anca y Aragón, 2007, p. 47).

En cuanto al sector en el que se concentran las empresas dirigidas por mujeres, el BIRF (2010) señala, que mientras los hombres se distribuyen entre una variedad de industrias, los negocios de las mujeres muestran una alta concentración en el comercio. Las empresas dirigidas por mujeres están sobre todo en el sector servicios, son de menor tamaño, tienen una posición tecnológica menos desarrollada y son mayoritariamente de tipo familiar (García, García y Madrid, 2012).

El perfil de las mujeres ejecutivas está más orientado a las personas, tiene características más sociables y con mayor tendencia a la cooperación. Además, ellas son más expresivas y cercanas, lo que les da un potencial importante a la hora de lograr un compromiso con la organización. Igualmente tienen la capacidad innata de pensar y actuar en muchas direcciones o temas al mismo tiempo y hacer que esto se potencie positivamente en su liderazgo como una herramienta importantísima (Attias, 2014). 
No obstante, a pesar de que la formación educativa ha representado un éxito social, no ha impactado en el escalafón o promoción profesional de las mujeres, ya que se continúa con paradigmas tradicionales que evitan el ingreso a puestos en concordancia a su nivel escolar (Gálvez, 2001). Las teorías que se han originado sobre la carrera profesional de las mujeres y su perfil de liderazgo (Sala, 2010), son:

- Modelo de asimilación: es el modelo de la power woman de los años ochenta, modelo de inspiración thatcherista según el cual para acceder a posiciones directivas las mujeres deben adoptar los patrones de dirección asociados al estilo masculino de dirección y renunciar a buscar un perfil propio (incluso en el modo de vestir).

- Modelo complementario: este modelo sostiene que hombres y mujeres presentan estilos diferentes de liderazgo que se adecuan a circunstancias diferentes. En ese sentido, algunos autores hablan del surgimiento de un liderazgo andrógino que disminuye los impactos de género y conjuga los mejores rasgos de los estilos masculinos y femeninos.

- Modelo del liderazgo femenino: presenta unas cualidades intrínsecamente superiores al masculino que se traducen en mejores resultados para las organizaciones. El liderazgo femenino se estructura en oposición al modelo patriarcal vertical y prima la colaboración y el networking por encima de la jerarquía.

- Modelo negacionista: niega la existencia de un modelo de liderazgo femenino.

Las demandas de la vida familiar hacen que las mujeres deban interrumpir más sus carreras y trabajen mayormente en jornadas parciales. Esto se traduce en menos años de experiencia y menos horas de trabajo por año, en comparación con los hombres, lo que ralentiza su progreso en las carreras, reduciendo sus ingresos y limitando su tiempo para construir redes profesionales, redes que pueden resultar decisivas para el avance de su trayectoria laboral (Eagly y Carli, 2007).

A pesar de la existencia del techo de cristal en las empresas, el ascenso progresivo de las mujeres en puestos directivos se convierte en una necesidad para las organizaciones que quieran competir con éxito en los mercados actuales. La necesidad de organizaciones heterogéneas que incorporen las ventajas de unos recursos humanos variados debería ser un objetivo en sí mismo que enriqueciera el modo de gestión de las empresas (Berenguer, Cerver, De la Torre, y Torcal, 2004, p. 147).

Powell y Eddleston (2011) sugieren que los hombres y las mujeres gestionen los negocios de forma distinta, por tanto, el sector, el tamaño, la edad organizacional, el carácter familiar, la junta directiva y el número de socios son herramientas de caracterización que conducen a marcar diferencias de gestión. 


\section{Método}

\section{Diseño de la investigación}

El presente estudio responde por sus características a una investigación con un enfoque cuantitativo transversal, al obtener la información en un tiempo específico y único. De alcance descriptivo, en la búsqueda de especificar las propiedades, las características y los perfiles de personas, grupos, comunidades, procesos, objetos o cualquier otro fenómeno (Hernández, Fernández y Baptista, 2014). Con el alcance descriptivo se identifican las características específicas de los perfiles y conductas de la mujer o la tendencia de este grupo en un ambiente empresarial y con cargos directivos.

\section{Población y muestra}

La población objeto de esta investigación son las y los gerentes directivos en mandos medios en las diferentes áreas de las empresas tequileras de Jalisco, incluyendo a todas aquellas empresas que cubrieran el requisito de contar con más de 30 trabajadores y de acuerdo con datos obtenidos en SIEM (Sistema de Información Empresarial Mexicana) se obtuvieron 12 empresas tequileras con ese requisito, resultando que solo 8 empresas permitieron aplicar la encuesta a sus directivos gerentes, con un total de 38 encuestados, 26 hombres y 12 mujeres.

\section{Selección del instrumento}

Para la obtención de la información se aplicó un instrumento integrado por dos apartados: en la primer parte se incluyeron 9 reactivos, de esos 3 son para caracterizar las empresas y 6 reactivos para obtener el perfil que tienen que ver con datos demográficos, tales como: edad, escolaridad, estado civil, número de hijos, etc. En el segundo apartado se integra la encuesta de Conductas del Liderazgo, instrumento creado y validado por Nader y Solano (2007), el cual consta de 18 reactivos y analiza 3 categorías sobre el desempeño del líder: las conductas orientadas hacia la tarea, las conductas orientadas hacia las relaciones y las conductas orientadas hacia el cambio.

Se evaluó la confiabilidad, aplicando el alfa de Cronbach para la encuesta del desempeño del líder, tomando en cuenta la muestra del estudio. Obteniéndose los resultados de confiabilidad que muestra la tabla 1 , donde se observa que la fiabilidad de los reactivos propuestos en el instrumento de medición resulta ser una correlación extremadamente alta, ya que la implementación del alfa de Cronbach arroja resultados bajo un rango estandarizado aceptado, el cual se encuentra entendido entre 0.8 y 0.55 , y cualquier resultado que se enfoque dentro de esos números se considera representativo de manera porcentual, lo cual indica que la congruencia de las preguntas permite favorecer su procesamiento en el software estadístico. 
Tabla 1. Alfa de Cronbach del cuestionario Conductas del Líder

\begin{tabular}{lcc}
\hline \multicolumn{1}{c}{ Dimensión } & Alfa de Cronbach & N. de elementos \\
\hline Conductas orientadas hacia la tarea & .788 & 6 \\
Conductas orientadas hacia las relaciones & .769 & 6 \\
Conductas orientadas hacia el cambio & .776 & 6 \\
\hline
\end{tabular}

Fuente. Elaboración propia.

El instrumentó se aplicó en los meses de marzo y abril de 2018, y una vez aplicadas las encuestas se integraron en una base de datos para su posterior procesamiento en el Paquete Estadístico para las Ciencias Sociales (SPSS), versión 11.

\section{Resultados}

Para la descripción de la caracterización de las empresas y de los encuestados, se presentan los resultados en donde se obtuvo información relevante con respecto a los siguientes aspectos: la antigüedad de las empresas tequileras encuestadas, en términos generales, la mayoría de ellas tienen una existencia de más de 10 años, y el $50 \%$ más de 20 años, según la tabla 2, identificándose que son empresas establecidas y con bastantes años de experiencia.

Tabla 2. Antigüedad de la empresa

\begin{tabular}{lcc}
\hline \multicolumn{1}{c}{ Rango de años } & No. de empresas & Porcentaje \\
\hline De 1 a 5 años & & \\
De 6 a 10 años & 2 & $25 \%$ \\
De 11 a 15 años & 2 & $25 \%$ \\
De 16 a 20 años & 4 & $50 \%$ \\
Más de 20 años & 8 & $100 \%$ \\
\hline Total & & \\
\hline
\end{tabular}

Fuente. Elaboración propia.

Con respecto al número de trabajadores que laboran en las empresas tequileras, de acuerdo con la tabla 3, la mayoría tiene más de 45 trabajadores, observándose que son empresas en su mayoría catalogadas de acuerdo con el Inegi (Instituto Nacional de Geografía e Informática, 2009) como medianas por el número de trabajadores.

Tabla 3. Número de trabajadores de las empresas

\begin{tabular}{lcc}
\hline \multicolumn{1}{c}{ Rango de trabajadores } & No. de empresas & Porcentaje \\
\hline De 30 a 35 trabajadores & & \\
De 36 a 40 trabajadores & 1
\end{tabular}




\begin{tabular}{lcc}
\hline \multicolumn{1}{c}{ Rango de trabajadores } & No. de empresas & Porcentaje \\
\hline De 46 a 50 trabajadores & 3 & \\
Más de 50 trabajadores & 4 & \\
Total & 8 & $100 \%$ \\
\hline
\end{tabular}

Fuente. Elaboración propia.

En la tabla 4, se muestra que el capital invertido en las empresas tequileras encuestadas más del 50\% es de procedencia nacional, evidenciándose que las empresas son en su mayoría de origen mexicano.

Tabla 4. Tipo de capital de las empresas

\begin{tabular}{|c|c|c|}
\hline Tipo de capital & No. de empresas & Porcentaje \\
\hline Nacional & 5 & $62.5 \%$ \\
\hline Extranjero & 1 & $12.5 \%$ \\
\hline Mixto & 2 & $25 \%$ \\
\hline Total & 8 & $100 \%$ \\
\hline
\end{tabular}

Fuente. Elaboración propia.

En relación con la edad de los encuestados en el caso de las mujeres la mayoría se ubica en edades de 45 en adelante y en los hombres la mayoría en edades de 55 años en adelante, resaltando que son de mayor edad los hombres que están en mandos medios con respecto a las mujeres que ocupan esos cargos (tabla 5).

Tabla 5. Edad de los gerentes

\begin{tabular}{lcccc}
\hline \multicolumn{1}{r}{ Rangos de edad } & Mujeres & Porcentaje & Hombres & Porcentaje \\
\hline De 35 a 45 años & 3 & $25 \%$ & 4 & $15.4 \%$ \\
De 46 a 55 años & 6 & $50 \%$ & 4 & $15.4 \%$ \\
\hline De 56 a 65 años & 3 & $25 \%$ & 12 & $46.1 \%$ \\
Más de 65 años & & & 6 & $23.1 \%$ \\
Total & 12 & $100 \%$ & 26 & $100 \%$ \\
\hline
\end{tabular}

Fuente. Elaboración propia.

El estado civil de los encuestados de acuerdo con la tabla 6, evidencia que más del $50 \%$ de las mujeres están casadas o en algún tipo de relación y en los hombres más del $80 \%$ está casado o en unión libre, permitiendo afirmar que los gerentes encuestados en su gran mayoría tienen algún tipo de relación de pareja. 
Tabla 6. Estado civil de los gerentes

\begin{tabular}{lcccc}
\hline \multicolumn{1}{c}{ Estado civil } & Mujeres & Porcentaje & Hombres & Porcentaje \\
\hline Soltero (a) & 2 & $16.7 \%$ & 3 & $11.5 \%$ \\
Casado (a) & 8 & $66.6 \%$ & 19 & $73.1 \%$ \\
Unión libre & 2 & $16.7 \%$ & 4 & $15.4 \%$ \\
Total & 12 & $100 \%$ & 26 & $100 \%$ \\
\hline
\end{tabular}

Fuente. Elaboración propia.

Se puede observar en la tabla 7, que las mujeres encuestadas tiene la gran mayoría de 2 y 3 hijos, y los hombres en su mayoría (50\%) de 4 a 5 hijos, reflejando que los hombres tienen más hijos con relación a las mujeres.

Tabla 7. Número de hijos de los gerentes

\begin{tabular}{lcccc}
\hline Rangos de número de hijos & Mujeres & Porcentaje & Hombres & Porcentaje \\
\hline Ningún hijo & 2 & $16.7 \%$ & 1 & $3.8 \%$ \\
\hline hijo & & & 2 & $7.7 \%$ \\
\hline 2 hijos & 4 & $33.3 \%$ & 5 & $19.2 \%$ \\
\hline 3 hijos & 5 & $41.7 \%$ & 7 & $26.9 \%$ \\
\hline 4 hijos & 1 & $8.3 \%$ & 6 & $23.1 \%$ \\
\hline 5 hijos & & & 3 & $11.6 \%$ \\
\hline Más de 5 hijos & 12 & $100 \%$ & 2 & $7.7 \%$ \\
\hline Total & & & 26 & $100 \%$ \\
\hline
\end{tabular}

Fuente. Elaboración propia.

La escolaridad de los gerentes encuestados de acuerdo con la tabla 8, muestra que las mujeres en su gran mayoría (más del 50\%), tienen maestría, algunas especialidad y otras doctorado, y los hombres se distribuyen la mayoría entre maestría y especialidad, observándose que un buen porcentaje de ellos (casi 35\%) solo tiene licenciatura y que en relación con las mujeres, ellas tienen en proporción a las encuestadas, un mayor porcentaje con doctorado (33.3\%), lo que revela que las mujeres se están preparando más que los hombres.

Tabla 8. Escolaridad de los gerentes

\begin{tabular}{lcccc}
\hline \multicolumn{1}{c}{ Escolaridad } & Mujeres & Porcentaje & Hombres & Porcentaje \\
\hline Licenciatura & 2 & $16.7 \%$ & 9 & $34.6 \%$ \\
Maestría & 4 & $33.3 \%$ & 8 & $30.8 \%$ \\
Especialidad & 2 & $16.7 \%$ & 8 & $30.8 \%$ \\
Doctorado & 4 & $33.3 \%$ & 1 & $3.8 \%$ \\
Total & 12 & $100 \%$ & 26 & $100 \%$ \\
\hline
\end{tabular}

Fuente. Elaboración propia. 
En términos de antigüedad en el puesto, las mujeres reflejaron en su mayoría que tienen menos de 10 años y solo una de ellas tiene más de 20 años, por otro lado, los hombres, al contrario, la mayoría tiene más de 15 años en el cargo actual, lo que permite deducir que los puestos gerenciales de mandos medios han sido cargos ocupados mayormente por hombres y las mujeres están actualmente buscando ascender a ellos, según se muestra en la tabla 9.

Tabla 9. Antigüedad de los gerentes en el puesto actual

\begin{tabular}{lcccc}
\hline \multicolumn{1}{c}{ Rango de años } & Mujeres & Porcentaje & Hombres & Porcentaje \\
\hline De 1 a 5 años & 4 & $33.3 \%$ & 5 & $19.2 \%$ \\
\hline De 6 a 10 años & 4 & $33.3 \%$ & 3 & $11.6 \%$ \\
\hline De 11 a 15 años & 2 & $16.7 \%$ & 4 & $15.4 \%$ \\
\hline De 16 a 20 años & 1 & $8.3 \%$ & 9 & $34.6 \%$ \\
\hline Más de 20 años & 1 & $8.3 \%$ & 5 & $19.2 \%$ \\
\hline Total & 12 & $100 \%$ & 26 & $100 \%$ \\
\hline
\end{tabular}

Fuente. Elaboración propia.

La forma de acceder a los puestos gerenciales de mandos medios (tabla 10) ha sido para las mujeres en un $66 \%$ por promoción y en los hombres también en su mayoría por el mismo proceso, aunque cabe resaltar que en el caso de los hombres un $23 \%$ de ellos tiene el cargo desde la constitución de la empresa, deduciendo que tienen ellos mayores formas de acceder al puesto actual que las mujeres.

Tabla 10. Forma de acceso al puesto actual de los gerentes

\begin{tabular}{lcccc}
\multicolumn{1}{c}{ Forma de acceso } & Mujeres & Porcentaje & Hombres & Porcentaje \\
\hline Proceso de selección & 4 & $33.3 \%$ & 8 & $30.8 \%$ \\
Promoción interna & 8 & $66.6 \%$ & 12 & $46.1 \%$ \\
Desde la constitución de la empresa & & & 6 & $23.1 \%$ \\
Total & 12 & $100 \%$ & 26 & $100 \%$ \\
\hline
\end{tabular}

Fuente. Elaboración propia.

Con la información recuperada de la segunda parte del cuestionario, se efectuó un estudio estadístico inferencial, aplicando la prueba t para muestras independientes. Obteniendo información relevante, al contrastar las 3 categorías de conductas del líder con la variable género, se evidencian diferencias estadísticamente significativas por género en la predilección por la implementación de prácticas orientadas hacia la tarea, variable donde las mujeres lograron mayor tendencia a diferencia de los hombres, y en las otras dos variables, la de conductas orientadas hacia las relaciones, las mujeres se quedaron por muy poco debajo de los hombres, y con respecto a la variable de prácticas con orientación hacia el cambio, los hombres tuvieron un promedio más alto, aunque no muy lejano de las mujeres, lo que demuestra en resumen, que en 
la presente investigación el estilo orientado hacia las tareas predominó en las mujeres, y cabe agregar, que las diferencias estadísticas encontradas son poco indicativas entre ambos sexos, en cuanto a las prácticas con orientación hacia las relaciones y en cuanto a las conductas orientadas hacia el cambio, donde las mujeres obtuvieron un promedio más bajo que los hombres. Lo que permite deducir que las mujeres al demostrar prácticas con orientación hacia las tareas o esquemas más gerenciales, pudieran estar influenciadas por una cultura paternalista y por la autoimagen que proyectan en su papel de líder (tabla 10).

Tabla 11. Comparación de las tres dimensiones de las conductas

del líder por el género

\begin{tabular}{|c|c|c|c|c|c|c|c|c|}
\hline Variables & $\begin{array}{c}\text { Niveles de } \\
\text { variables }\end{array}$ & & $\mathbf{N}$ & M & DE & gl & t & p \\
\hline \multirow{2}{*}{$\begin{array}{l}\text { Conductas del líder } \\
\text { orientadas hacia } \\
\text { la tarea }\end{array}$} & Masculino & & 26 & 24.70 & 3.45 & \multirow[t]{2}{*}{36} & \multirow[t]{2}{*}{-1.61} & \multirow[t]{2}{*}{.34} \\
\hline & Femenino & & 12 & 28.06 & 2.20 & & & \\
\hline \multirow{2}{*}{$\begin{array}{l}\text { Conductas del líder } \\
\text { orientadas hacia las } \\
\text { relaciones }\end{array}$} & Masculino & & 26 & 27.38 & 2.25 & \multirow[t]{2}{*}{36} & \multirow[t]{2}{*}{-3.41} & \multirow[t]{2}{*}{$* .01$} \\
\hline & Femenino & & 12 & 27.02 & 2.66 & & & \\
\hline \multirow{2}{*}{$\begin{array}{l}\text { Conductas del líder } \\
\text { orientadas hacia el } \\
\text { cambio }\end{array}$} & Masculino & & 26 & 26.09 & 3.07 & \multirow{2}{*}{36} & \multirow{2}{*}{-2.92} & \multirow{2}{*}{.13} \\
\hline & Femenino & 12 & & 25.48 & 3.25 & & & \\
\hline
\end{tabular}

\section{Hallazgos y discusiones}

El perfil de la mujer directiva en puestos gerenciales corresponde a una mujer casada $(66.6 \%)$, entre 46 y 55 años $(50 \%)$, con tres hijos $(41.7 \%)$ y tiene una formación superior, orientada a las ciencias empresariales y económicas, dentro de esta resalta que un $33.3 \%$ ha cursado maestría y un $33.3 \%$ ha realizado además un doctorado. Con una antigüedad en el puesto actual, la mayoría, en promedio de 1 a 10 años $(66.6 \%)$. Y la forma de acceder al puesto actual en la mayoría ha sido por promoción interna $(66.6 \%)$.

Dentro de las conductas del líder, las mujeres encuentran más motivación a las tareas, y han obtenido los mejores promedios, significando que sus aptitudes están mejor encaminadas a la responsabilidad directiva y a la ejecución de actividades. 


\section{Conclusiones}

La incorporación de las mujeres al mercado de trabajo ha constituido uno de los fenómenos más característicos en la actualidad, fenómeno generalizado que se verifica en los países de nuestro entorno geográfico, económico y cultural, observándose un aumento significativo y constante, tanto de la participación laboral de las mujeres como de su nivel formativo. Sin embargo, esta incorporación masiva de las mujeres al mercado laboral no se ha visto aún traducida en un incremento proporcional de mujeres en posiciones de poder y puestos laborales con capacidad de decisión.

Con los resultados de esta investigación se coincide con los estudios de Eagly y Johnson (1990) y Camps, Pérez y Martínez (2010), quienes afirman que hombres y mujeres realizan un estilo de líder centrado en las tareas, cuando representan un papel en la organización conforme a su género. Al demostrar que las mujeres liderean con estilos más democráticos y participativos, mientras que los hombres lo realizan de forma más autocrática o directiva.

El estudio ha demostrado que en el ámbito empresarial tequilero de Jalisco, aún prevalecen formas de gerencia restringida y menos adecuada con la actualidad empresarial, ya que dichas prácticas permiten y promueven inequidad en el ingreso de hombres y mujeres con el mismo nivel educativo a puestos directivos y gerenciales que implican mayor compromiso.

En conclusión, en el ambiente económico global en el que se desempeñan las organizaciones tequileras es indispensable el cambio organizacional que implique la pluralidad de género y se aprovechen las diferencias relacionadas a esta. En ese sentido, será importante para la competitividad de la empresa transitar hacia prácticas de desempeño incluyentes y colaborativas, que integren las bondades relacionadas con el liderazgo centrado en las tareas.

Finalmente, con los resultados obtenidos en el presente estudio, se evidencia que existen considerables limitaciones para el ingreso y desarrollo de las mujeres dentro de las organizaciones; desde su función social y lo que va implícito como obligaciones inherentes, afectando en la percepción que se tiene de su desempeño. De ahí que es necesario, el construir un cambio cultural en las empresas orientado a ofrecer mayores y mejores oportunidades de desarrollo y crecimiento a la mujer dentro del entorno, que le permita demostrar sus capacidades, siendo un imperativo la creación de mecanismos o políticas que propicien un ambiente adecuado para la equidad de género en cargos directivos. 


\section{Referencias}

Attías, A. (2014). Mujeres en el poder. El liderazgo femenino es el crecimiento del siglo XXI. Clarín. Entre mujeres. Recuperado de https://www.clarin.com/trabajo/ trabajo-liderazgo_femenino-mujeres-lideres-crecimiento_0_rJwTX5Kwmx.html

Barberá, E., Ramos, A., y Candela, C. (2011). Laberinto de cristal en el liderazgo de las mujeres. Psicothema, 23(2), 173-179.

Berenguer, G., Cerver, E., De la Torre, A., y Torcal, R. (2004). El estilo directivo de las mujeres y su influencia sobre la gestión del equipo de trabajo en las cooperativas valencianas. CIRIEC. Revista de economía pública, social y cooperativa, 50, 123 149.

BIRF. (2010). Mujeres empresarias barreras y oportunidades en el Sector Privado formal en América Latina y el Caribe. Washington, D.C.: Banco Internacional de Reconstrucción y Fomento, Banco Mundial.

Charlo, M., y Núñez, M. (2012). La mujer directiva en la gran empresa española: perfil, competencias y estilos de dirección. Estudios Gerenciales, 124, 87-105.

Camps, V., Pérez, J., y Martínez, M. (2010). Comparación por género de los estilos de liderazgo en una muestra de gerenciales en Puerto Rico. Puertorriqueña de Psicología, 21, 113-132.

Clancy, S. (2007). ¿Por qué no hay más mujeres en la cima de la escala corporativa: debido a estereotipos, a diferencias biológicas o a escogencias personales? Academia Latinoamericana de Administración, 38, 1-8.

Coelho, A. (2007). Eficiencia colectiva y upgrading en el cluster del tequila. Análisis Económico, 22(49), 169-194.

Contreras, F., Pedraza, J., y Mejía, X. (2012). La mujer y el liderazgo empresarial. Diversitas - Perspectivas en psicología, 8(1), 183-193.

Cuadrado, I.; Navas, M., y Molero, F. (2004). El acceso de las mujeres a puestos directivos: género, contexto organizacional y estilos de liderazgo. Psicología General y Aplicada, 57, 181-192.

Cuadrado, I., y Morales, J. (2007). Algunas claves sobre el techo de cristal en las organizaciones. Psicología del Trabajo y de las Organizaciones, 23(2), 183-202.

De Anca, C., y Aragón, S. (2007). La mujer directiva en España: catalizadores e inhibidores en las decisiones de trayectoria profesional. Academia Latinoamericana de Administración, 38, 45-63.

Eagly, A., y Carli, L. (2007). Women and the Labyrinth of Leadership. Harvard Business Review, 85, 63-71. 
Eagly, A. y Johonson, B. (1990). Gender and leadership style: A meta-analysus. Psychological Bulletin, 108(2), 233-256.

Gálvez, T. (2001). Aspectos económicos de la equidad de género. Unidad mujer y desarrollo, No. 35. CEPAL. Recuperado de http://www.eclac.cl/publicaciones/ $\mathrm{xml} / 4 / 7374 / 1 \mathrm{cl} 1561 \mathrm{e} . \mathrm{pdf}$

García, B., y De Oliveira, O. (2006). Las familias en el México metropolitano: Visiones femeninas y masculinas. Centro de Estudios Demográficos Urbanos y Ambientales, El Colegio de México. México.

García, M., García, D., y Madrid, A. (2012). Caracterización del comportamiento de las Pymes según el género del gerente: un estudio empírico. Cuadernos de Administración, 28(47), 37-52.

García, M., González, C. H., y Murillo, G. (2017). Características de las pymes según la gerencia sea hombre o mujer: caso empírico de Cali. Revista EAN, 82, 147-164 doi: 10.21158/01208160.n82.2017.1648

García, M., Salas, L. y Gaviria, E. (2017). Estilos de liderazgo de hombres y mujeres en las pymes. $A D$-minister. $31,25-46$.

Hernández, S. R., Fernández, C. C., y Baptista, P. (2014). Metodología de la investigación. (5 $5^{\mathrm{a}}$ ed.). México: Mc Graw-Hill.

Instituto Nacional de Estadística y Geografía - Inegi (2003). Población ocupada según sexo, 1950-2000. Recuperado de: http://www.inegi.gob.mx/est/contenidos/ espanol/tematicos/mediano/anu.asp? $\mathrm{t}=\mathrm{mtra} 08 \& \mathrm{c}=3599$

Instituto Nacional de Estadística y Geografía - INEGI (2009). Micro, pequeñas y medianas empresas. Recuperado de:

www.inegi.org.mx/est/contenidos/espanol/.../pdf/Mono_Micro_peque_mediana.pdf.

Macías, A. (1997). Organización de la Industria del Tequila. Carta Económica Regional, 9 (54), 3-11, doi: 10.32870/cer.v0i54.7643

Moncayo, B. y Zuluaga, D. (2015) Liderazgo y género: barreras de mujeres directivas en la academia. Pensamiento \& Gestión, (39), 142-177.

Nader, M., \& Solano, A. C. (2007). Influencia de los valores sobre los estilos de liderazgo: Un análisis según el modelo de liderazgo transformacionaltransaccional de Bass. Universitas Psychologica, 6(3), 689-698.

ONU (2005). Objetivos de Desarrollo del Milenio: Una mirada desde América Latina y el Caribe. Recuperado de http://www.eclac.org/publicaciones/xml/1/21541/ $\operatorname{lcg} 2331$ e.pdf 
Powell, G. N. y Eddleston, K. A. (2011). Work family enrichment and entrepreneurial success: Do female entrepreneurs benefit most? The Academy of Management, $32,(3), 747-760$.

Sala, M. (2010). Mujer y liderazgo. Unidad de Conocimiento. Recuperado de https:// factorhuma.org/attachments_secure/article/8259/dona_i_lideratge_cast.pdf

Selva, C., Sahagún, M., y Pallarés, S. (2011). Estudios sobre trayectoria profesional y acceso de la mujer a cargos directivos: Un análisis bibliométrico. Psicología del Trabajo y de las Organizaciones, 27(3), 227-242.

SIEM. Sistema de Información Empresarial Mexicana. www.siem.gob.mx 